Homology, Homotopy and Applications, vol.19(2), 2017, pp.89-110

\title{
EXTENDING HOMOTOPY THEORIES ACROSS ADJUNCTIONS
}

\author{
NICK GURSKI, NILES JOHNSON AND ANGÉLICA M. OSORNO
}

(communicated by Emily Riehl)

\begin{abstract}
Constructions of spectra from symmetric monoidal categories are typically functorial with respect to strict structurepreserving maps, but often the maps of interest are merely lax monoidal. We describe conditions under which one can transport the weak equivalences from one category to another with the same objects and a broader class of maps. Under mild hypotheses this process produces an equivalence of homotopy theories. We describe examples including algebras over an operad, such as symmetric monoidal categories and $n$-fold monoidal categories; and diagram categories, such as $\Gamma$-categories.
\end{abstract}

\section{Introduction}

The classifying space functor from categories to topological spaces provides a way of constructing spaces with certain algebraic structure. Of particular importance are infinite loop space machines, which construct spectra out of structured categories such as symmetric monoidal categories [Sta71, Qui73, May74, Seg74, Wal85, EM06, May09, Oso12]. The discussion of the functoriality of these constructions is somewhat nuanced due to the range of possible morphisms one might choose. These morphisms differ in strength, the degree to which the underlying functors of structured categories preserve the structure.

It is often the case that such machines are obviously functorial with respect to maps that strictly preserve the ambient structure. This is the case, for example, for the operadic machine and maps of symmetric monoidal categories. The maps that arise in practice however - for example, the functors of module categories induced by a morphism of commutative rings - are typically not strict, but strong or merely lax, meaning that they preserve monoidal structure up to coherent isomorphism or merely coherent morphism. One way to handle such variation is to construct variant machinery for each type of morphism and prove that the corresponding constructions are equivalent. This allows one to prove general theorems about the strict case, for example, Segal machinery and strict maps of $\Gamma$-categories, but apply them to the more broadly useful strong or lax case, for example, Segal machinery and lax maps. Such

Received October 4, 2016, revised December 7, 2016; published on September 6, 2017.

2010 Mathematics Subject Classification: 55U35, 18C20, 19D23, 18A25, 18 D50.

Key words and phrases: lax map, strict map, 2-monad.

Article available at http://dx.doi.org/10.4310/HHA.2017.v19.n2.a6

Copyright (C) 2017, Nick Gurski, Niles Johnson and Angélica M. Osorno. Permission to copy for private use granted. 
an approach appears in a number of places in the literature, for example, in [Man10, $\S 3]$.

In this paper we consider a more systematic approach: a direct comparison of the homotopy theories arising from structured categories and maps of various strength. For this purpose, we discuss homotopy theory in the generality of relative categories. A relative category is merely a category $\mathcal{C}$ equipped with a subcategory $\mathcal{W}$ containing all of the objects. The morphisms in this subcategory then play the role of weak equivalences. A pair $(\mathcal{C}, \mathcal{W})$ presents a homotopy theory [Rez01, BK12], and such a presentation neatly hides, but crucially still retains, higher homotopical information, such as mapping spaces, that is not present in the bare homotopy category.

The central problem we address in this paper may then be described as follows.

Problem. For a homotopy theory $(\mathcal{C}, \mathcal{W})$, give criteria for enlarging the class of morphisms in $\mathrm{C}$ to give a new category $\mathrm{C}^{\prime}$ with a larger class of weak equivalences $\mathcal{W}^{\prime}$ such that the inclusion $(\mathcal{C}, \mathcal{W}) \hookrightarrow\left(\mathrm{C}^{\prime}, \mathcal{W}^{\prime}\right)$ is an equivalence of homotopy theories.

In other words, how can we replace the morphisms in $\mathcal{C}$ with more flexible ones without changing the homotopy theory? The advantages of such a strategy are well-known: the smaller class of morphisms is likely more amenable to abstract manipulation, while the larger class will often arise in examples of interest.

Our first main result, Theorem 1.11, gives general conditions under which one can extend the class of weak equivalences via an adjunction

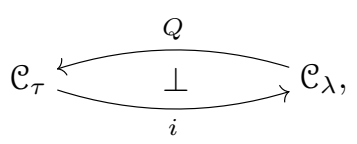

with $i$ the identity on objects. Moreover, we prove that this extension is unique and that the resulting homotopy theories are equivalent. This should be seen as the relative-categorical analogue of a very strong kind of transferred model structure for Quillen model categories (see, for example, [Cra95]).

Our second main result, Theorem 2.8 , takes $\mathcal{C}_{\tau}$, respectively $\mathcal{C}_{\lambda}$, to be categories of algebras and strict, respectively lax, maps for a 2 -monad $T$ on a 2 -category $\mathcal{K}$. In particular, $T$ may be the 2 -monad on $\mathcal{K}=\mathcal{C} a t$ whose algebras are symmetric monoidal categories. This special case provides an enhancement of previous work in the case of symmetric monoidal categories: Thomason and Mandell show that the corresponding homotopy categories are equivalent after localizing stable equivalences [Tho95, Lemma 1.9.2] and weak equivalences [Man10, Theorem 3.9], respectively. Many other examples of interest arise in this way, and we describe a number of them in detail.

We choose the framework of relative categories, rather than Quillen model structures, as many of the categories we encounter are not well-behaved enough to construct model structures. For example, one variant of our results (see Theorem 2.15) shows that the homotopy theory of symmetric monoidal categories using strict symmetric monoidal functors and stable equivalences extends uniquely to an equivalent homotopy theory on the category of symmetric monoidal categories using lax symmetric monoidal functors. While it is straightforward to define compatible weak equivalences in these categories, the latter category is neither complete nor cocomplete so, in particular, constructing a model structure via the small object argument is not possible. 


\section{Outline}

In Section 1 we recall basic notions of relative categories and give our first main result regarding equivalences of homotopy theories for strict and lax maps.

In Section 2 we apply the results of Section 1 to the different morphism variants for algebras over a 2-monad using the factorization system techniques of Bourke and Garner [BG16b]. We then go on to give the following examples: symmetric monoidal categories and $n$-fold monoidal categories (Section 2.1); categories with group actions (Section 2.2); and $\Gamma$-categories or $\Gamma$-2-categories (Section 2.3). In each case we discuss interesting map variants, classes of weak equivalences, and explicitly state the resulting equivalence of homotopy theories.

In Section 3 we recall Bourke's theory of 2-dimensional monadicity [Bou14]. We use this theory to recognize some naturally-occurring morphisms as the lax algebra morphisms for various 2-monads, thus completing the proofs required for some of the examples in Section 2.

\section{Acknowledgments}

The first author was supported by EPSRC EP/K007343/1. The third author was partially supported by a grant from the Simons Foundation (\#359449, Angélica Osorno). The authors thank Mark Behrens for a number of useful comments.

\section{Adjunctions creating weak equivalences}

In this section we develop the fundamental machinery to extend a notion of weak equivalence from a given category to one with the same set of objects and a larger class of morphisms. We give conditions which guarantee that this extension yields an equivalence of homotopy theories.

To begin, we recall the elementary notions of relative categories. For more details, see [DK80, Rez01, BK12].

Definition 1.1. A relative category is a pair $(\mathcal{C}, \mathcal{W})$ in which $\mathcal{C}$ is a category and $\mathcal{W}$ is a subcategory of $\mathcal{C}$ containing all of the objects. A relative functor $F:(\mathcal{C}, \mathcal{W}) \rightarrow$ $\left(\mathrm{C}^{\prime}, \mathcal{W}^{\prime}\right)$ is a functor $F: \mathcal{C} \rightarrow \mathcal{C}^{\prime}$ such that $F$ restricts to a functor $\mathcal{W} \rightarrow \mathcal{W}^{\prime}$. A relative adjunction is an adjunction

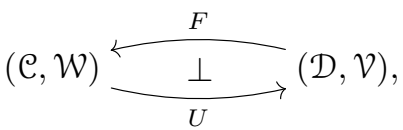

where $F$ and $U$ are relative functors.

Definition 1.3. A category with weak equivalences is a relative category $(\mathcal{C}, \mathcal{W})$, where $\mathcal{W}$ contains all isomorphisms and satisfies the 2-out-of-3 property. We informally refer to a category with weak equivalences as a homotopy theory.

Definition 1.4. Let $(\mathcal{C}, \mathcal{W})$ and $(\mathcal{D}, \mathcal{V})$ be categories with weak equivalences. We say a functor $F: \mathcal{C} \rightarrow \mathcal{D}$ creates weak equivalences if for each morphism $f$ of $\mathcal{C}, f \in \mathcal{W}$ if and only if $F f \in \mathcal{V}$. 
We now recall the definition of equivalence between homotopy theories [Rez01]. This notion is equivalent to the requirement that the induced map on hammock localizations be a DK-equivalence, and implies that the induced map on categorical localizations is an equivalence [BK12].

Definition 1.5. A relative functor $F:(\mathcal{C}, \mathcal{W}) \rightarrow(\mathcal{D}, \mathcal{V})$ is an equivalence of homotopy theories if, in the complete Segal space model structure, the induced map on fibrant replacements of classification diagrams is a weak equivalence.

Convention 1.6. Given a collection of weak equivalences, $\mathcal{W}$, and a natural transformation $\eta$, we say that $\eta$ is a weak equivalence and write $\eta \in \mathcal{W}$ if each component of $\eta$ is in $\mathcal{W}$.

For reference, we record the following observation. Further discussion appears in [GJO17, 2.9].

Lemma 1.7. A relative adjunction whose unit and counit are weak equivalences induces an equivalence of homotopy theories.

This lemma motivates the following definition.

Definition 1.8. We say that a relative adjunction is an adjoint equivalence of homotopy theories if the components of its unit and counit are weak equivalences.

We are interested in the interplay between different types of morphisms between given objects, and thus make the following definition.

Definition 1.9. A map extension of a category $\mathcal{C}_{\tau}$ is an inclusion $i: \mathcal{C}_{\tau} \hookrightarrow \mathcal{C}_{\lambda}$ which is the identity on objects. We refer to the morphisms of $\mathcal{C}_{\tau}$ as tight, and those of $\mathcal{C}_{\lambda}$ as loose.

For example, one might take the tight maps between monoidal categories to be the strict monoidal functors and the loose maps to be the lax monoidal functors, the oplax monoidal functors, the strong monoidal functors, etc. A map extension is a special case of what [LS12] call an $\mathscr{F}$-category.

Definition 1.10. Let

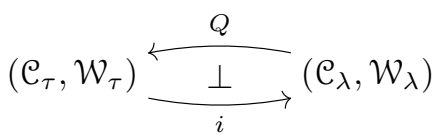

be a relative adjunction. We say $Q \dashv i$ creates weak equivalences if both $i$ and $Q$ create weak equivalences.

It is generally not the case that a relative adjunction creates a class of weak equivalences in this sense. We will, however, describe useful hypotheses which guarantee this in a number of interesting examples.

Theorem 1.11. Let $\left(\mathcal{C}_{\tau}, \mathcal{W}_{\tau}\right),\left(\mathcal{C}_{\lambda}, \mathcal{W}_{\lambda}\right)$ be categories with weak equivalences and let

$$
\mathrm{C}_{\tau} \stackrel{i}{\rightarrow} \mathcal{C}_{\lambda}
$$

be a map extension. Assume there is a left adjoint $Q \dashv i$ with counit $\varepsilon$ and unit $\eta$.

Then the following are equivalent: 
1. $i$ creates weak equivalences and $\varepsilon \in \mathcal{W}_{\tau}$,

2. $i$ creates weak equivalences and $\eta \in \mathcal{W}_{\lambda}$,

3. $Q$ creates weak equivalences and $\varepsilon \in \mathcal{W}_{\tau}$.

Moreover, these conditions imply the following:

4. $Q$ creates weak equivalences and $\eta \in \mathcal{W}_{\lambda}$.

Consequently,

$$
\left(\mathcal{C}_{\tau}, \mathcal{W}_{\tau}\right) \stackrel{Q}{\stackrel{\perp}{i}}\left(\mathcal{C}_{\lambda}, \mathcal{W}_{\lambda}\right)
$$

is an adjoint equivalence of homotopy theories.

Proof. With $Q \dashv i$, we have the following triangle identities for $A \in \mathcal{C}_{\tau}$ and $B \in \mathcal{C}_{\lambda}$.
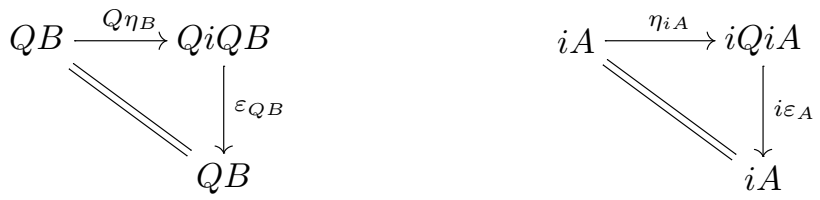

We first show $(1) \Leftrightarrow(2)$ and $(3) \Rightarrow(4)$ : If $i$ creates weak equivalences and $\varepsilon_{A} \in \mathcal{W}_{\tau}$, then $i \varepsilon_{A} \in \mathcal{W}_{\lambda}$ and therefore $\eta_{i A} \in \mathcal{W}_{\lambda}$ by the 2 -out-of-3 property. But since $i$ is the identity on objects, we have that $\eta \in \mathcal{W}_{\lambda}$. Conversely, $\eta \in \mathcal{W}_{\lambda}$ implies each $i \varepsilon_{A} \in \mathcal{W}_{\lambda}$ and therefore $\varepsilon \in \mathcal{W}_{\tau}$ since $i$ creates weak equivalences. Likewise, if $Q$ creates weak equivalences and $\varepsilon \in \mathcal{W}_{\tau}$, then $\eta \in \mathcal{W}_{\lambda}$.

Now we show (1) and (2) together imply (3). To do so, we need only show that $Q$ creates weak equivalences. Let $f: A \rightarrow B$ in $\mathcal{C}_{\lambda}$. The naturality square for $\eta$ at $f$ together with the 2-out-of-3 property imply that $f \in \mathcal{W}_{\lambda}$ if and only if $i Q f \in \mathcal{W}_{\lambda}$. Therefore, since $i$ creates weak equivalences, so does $Q$.

A similar argument using naturality of $\varepsilon$ shows (3) and (4) together imply (1).

Note. We emphasize that condition (4) does not generally imply the others.

Remark 1.12. In practice, we have a notion of weak equivalences in $\mathcal{C}_{\tau}$ and want to extend this notion to the more general maps in $\mathcal{C}_{\lambda}$ in a conservative way: we do not want a tight map to become a weak equivalence when considered as a loose map. The fact that conditions (1) and (3) in Theorem 1.11 are equivalent means that whenever $\varepsilon$ is a weak equivalence we can achieve this by creating $\mathcal{W}_{\lambda}$ via $Q$.

The same reasoning above, applied to different triangle identities, yields the following version of Theorem 1.11 when $i$ has a right adjoint. We will not use this version, but include it for completeness.

Theorem 1.13. Let $\left(\mathcal{C}_{\tau}, \mathcal{W}_{\tau}\right),\left(\mathcal{C}_{\lambda}, \mathcal{W}_{\lambda}\right)$ be categories with weak equivalences and let $\mathcal{C}_{\tau} \stackrel{i}{\rightarrow} \mathcal{C}_{\lambda}$ be a map extension. Assume there is a right adjoint $i \dashv Q$ with counit and unit $\varepsilon$ and $\eta$, respectively. The following are equivalent: 
1. $i$ creates weak equivalences and $\eta \in \mathcal{W}_{\tau}$,

2. $i$ creates weak equivalences and $\varepsilon \in \mathcal{W}_{\lambda}$,

3. $Q$ creates weak equivalences and $\eta \in \mathcal{W}_{\tau}$.

Moreover, these conditions imply the following:

4. $Q$ creates weak equivalences and $\varepsilon \in \mathcal{W}_{\lambda}$.

Consequently,

$$
\left(\mathfrak{C}_{\tau}, \mathcal{W}_{\tau}\right) \stackrel{Q}{\stackrel{\mathrm{T}}{i}}\left(\mathfrak{C}_{\lambda}, \mathcal{W}_{\lambda}\right)
$$

is an adjoint equivalence of homotopy theories.

The next result shows that if weak equivalences in the category of tight maps are detected via some underlying data, then the same is true for the loose maps. This is the most common situation in examples of interest.

Theorem 1.14. Assume the hypotheses and any of the equivalent statements of Theorem 1.11. Furthermore, let $(\mathcal{K}, \mathcal{V})$ be a category with weak equivalences with a commutative triangle of underlying categories as below.

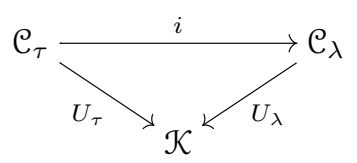

Then $U_{\tau}$ creates $\mathcal{W}_{\tau}$ if and only if $U_{\lambda}$ creates $\mathcal{W}_{\lambda}$.

Proof. One implication is obvious: if $U_{\lambda}$ creates weak equivalences then so does $U_{\tau}$. Now for the converse assume that $U_{\tau}$ creates weak equivalences. We first show that $U_{\lambda} \eta \in \mathcal{V}$. Applying $U_{\lambda}$ to one of the triangle identities shows that

$$
\operatorname{id}_{U_{\tau} A}=U_{\lambda} i \varepsilon_{A} \circ U_{\lambda} \eta_{i A}=U_{\tau} \varepsilon_{A} \circ U_{\lambda} \eta_{i A},
$$

so 2-out-of-3, the fact that $i$ is the identity on objects, and the assumption that $U_{\tau}$ creates weak equivalences shows $U_{\lambda} \eta \in \mathcal{V}$.

Now let $f$ be a morphism of $\mathcal{C}_{\lambda}$. Naturality of $\eta$ shows that $\eta \circ f=i Q f \circ \eta$. Applying $U_{\lambda}$ to this equation gives

$$
U_{\lambda} \eta \circ U_{\lambda} f=U_{\lambda} i Q f \circ U_{\lambda} \eta=U_{\tau} Q f \circ U_{\lambda} \eta .
$$

By Theorem 1.11, $Q$ creates weak equivalences. So $f \in \mathcal{W}_{\lambda}$ if and only if $U_{\tau} Q f \in \mathcal{V}$ and, so the result follows by 2 -out-of-3 and $U_{\lambda} \eta \in \mathcal{V}$.

Definition 1.15. We say that $Q \dashv i$ is an adjoint equivalence of homotopy theories $\operatorname{over}(\mathcal{K}, \mathcal{V})$ and write

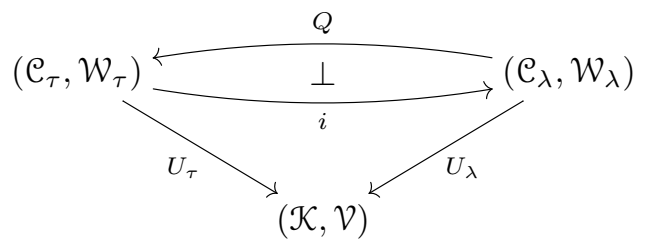


to mean:

1. $Q \dashv i$ is an adjoint equivalence of homotopy theories,

2. $U_{\lambda} \circ i=U_{\tau}$, and

3. both $U_{\lambda}$ and $U_{\tau}$ create weak equivalences.

Note in particular that the triangle involving $Q$ does not generally commute.

\section{Applications to algebras over 2-monads}

We will apply the results on homotopy theories in the previous section to various categories of algebras over 2-monads. We assume the reader is familiar with basic 2-monad theory as developed in, e.g., [KS74, BKP89].

Throughout this section we let $\mathcal{K}$ be a complete and cocomplete 2-category (in the Cat-enriched sense), and let $T: \mathcal{K} \rightarrow \mathcal{K}$ be a 2 -monad. Let $T$ - $\mathcal{A l g}_{s}$ denote the 2 category whose 0 -cells are $T$-algebras, 1 -cells are strict algebra maps, and 2-cells are $T$-algebra transformations. There are also notions of lax, oplax and pseudo algebra maps, which are, respectively, the 1 -cells in the 2 -categories $T$ - $\mathcal{A} \mathcal{L}_{l}, T-\mathcal{A} \mathscr{I}_{\text {op }}$, and $T-\mathcal{A l g}_{p s}$.

In examples, $T$ might describe (symmetric) monoidal structures, $n$-fold monoidal structures, diagrams in a 2-category, or $G$-equivariant structures for a group $G$. In the monoidal case, the four kinds of maps are:

- strict monoidal, with axioms like $F(x) \otimes F(y)=F(x \otimes y)$;

- lax monoidal, with additional data like $F(x) \otimes F(y) \rightarrow F(x \otimes y)$, subject to new coherence axioms;

- oplax monoidal, with additional data like $F(x \otimes y) \rightarrow F(x) \otimes F(y)$, subject to the "backwards" version of the lax axioms; and

- strong monoidal (pseudo algebra maps), with additional data like $F(x) \otimes F(y) \cong$ $F(x \otimes y)$, once again subject to new coherence axioms.

Definition 2.1 (2-monadic). A 2-functor is called 2-monadic if it is monadic in the Cat-enriched sense.

Let $U_{\omega}: T-\mathcal{A} \mathcal{L}_{\omega} \rightarrow \mathcal{K}$ denote any of the functors which give the underlying objects and morphisms, where $\omega$ denotes any of $s, l$, op, or $p s$. The functor $U_{s}$ is then 2-monadic, and any 2-monadic functor is of this form (up to 2-equivalence of 2categories); in particular, one should note that 2-monadicity does not capture the structure of any of the non-strict variants.

The Cat-enriched monadicity theorem [Dub70] gives three essential conditions which imply that a 2-functor $U: X \rightarrow Y$ is 2-monadic. First, it must have a left 2adjoint. Second, it must be conservative (see below). Third, $X$ must have, and $U$ must preserve, certain coequalizers.

Definition 2.2 (Conservative). A functor is called conservative if it reflects isomorphisms.

Definition 2.3 (Accessible). A functor is accessible if it preserves $\kappa$-filtered colimits for some regular cardinal $\kappa$. A monad is called accessible if its underlying functor is accessible. 
Theorem 2.4 ([BKP89]). If $T$ is an accessible 2-monad on a complete and cocomplete 2-category $\mathcal{K}$, then the inclusion

$$
i: T-\mathcal{A l g}_{s} \hookrightarrow T-\mathcal{A l g}_{l}
$$

has a left 2-adjoint $Q$.

Remark 2.5. The above result holds when lax is replaced with oplax or pseudo.

Bourke and Garner show in [BG16b] that $Q$ arises from an algebraic weak factorization system using the class of lalis. Here we are required to use the additional power of an algebraic weak factorization system over the more traditional weak factorization systems. Algebraic refers to additional structure we require our factorization system to possess. Instead of having left and right classes of maps satisfying factorization and lifting axioms, we have a functorial factorization $f \mapsto R f \circ L f$ equipped with the structure of a monad on the functor $R$ and a comonad on the functor $L$. The coalgebras for $L$ play the role of left maps, and the algebras for $R$ play the role of right maps. A (co)algebra structure is just that: additional structure. Thus we talk about right map structures on a given morphism, meaning a choice of algebra structure for the monad $R$. While the proofs of the results quoted here depend heavily on this extra algebraic structure, the theory of algebraic weak factorization systems can be taken as a black box for our purposes. For further reading, see [GT06, BG16a, BG16b].

Definition 2.6. A left-adjoint left-inverse, or lali, in $\mathcal{K}$ is an adjunction $(f \dashv g$, $\varepsilon: f g \Rightarrow \mathrm{id}, \eta: \mathrm{id} \Rightarrow g f)$ such that $\varepsilon$ is the identity.

Proposition 2.7 ([BG16b]). Let $T$ be an accessible 2-monad on a complete and cocomplete 2-category $\mathcal{K}$.

1. There is an algebraic weak factorization system on the underlying category of $\mathcal{K}$ such that a right map structure on a map $f$ is a lali structure $(f \dashv g, \varepsilon=\mathrm{id}, \eta)$.

2. There is an algebraic weak factorization system on the underlying category of $T$ - $\mathcal{A l g}_{s}$ such that a right map structure on a strict algebra map $f: A \rightarrow B$ in $T-\mathcal{A l g}_{s}$ is a lali structure on the underlying 1-cell in $\mathcal{K}$.

3. The inclusion $i$ has a left adjoint $Q$

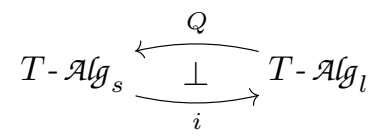

and the counit $\varepsilon$ of this adjunction has a right map structure as in (2).

We combine the previous result with the theory of Section 1 to prove the following. This is the theorem we use most frequently in examples.

Theorem 2.8. Let $T$ be an accessible 2-monad on a complete and cocomplete 2category $\mathcal{K}$. Let $\mathcal{W}_{s}$ be a collection of 1-cells which make the underlying 1-category of $T$ - $\mathcal{A l g}_{s}$ a category with weak equivalences and assume $\mathcal{W}_{s}$ contains all 1-cells $f$ such that $U_{s} f$ admits a lali structure. Then there exists a left adjoint $Q$ and a unique 
collection of 1-cells $\mathcal{W}_{l}$ created by $Q \dashv i$. Consequently,

$$
\left(T-\mathcal{A l g}_{s}, \mathcal{W}_{s}\right) \stackrel{Q}{\frac{\perp}{i}}\left(T-\mathcal{A l g}_{l}, \mathcal{W}_{l}\right)
$$

establishes an adjoint equivalence of homotopy theories.

Proof. This follows by combining Theorem 1.11 and Proposition 2.7.

Remark 2.9. There is a version of this theory that works with oplax morphisms instead of lax ones, and the algebraic weak factorization system involved uses ralis (right adjoint, left inverse) for its right maps instead of lalis. Alternatively, there is a pseudo-strength version, using pseudomorphisms, and the corresponding algebraic weak factorization system is that for retract equivalences. See [BG16b] for more details. In each case we have a corresponding version of Proposition 2.7 and Theorem 2.8.

\subsection{Monads on $\mathcal{K}=$ Cat}

Let $\mathcal{K}=$ Cat and let $T$ be any accessible 2 -monad, for example, the 2 -monad arising from an operad. Let $(\mathrm{Cat}, \mathcal{V})$ be any weak equivalence structure for which $\mathcal{V}$ contains all adjunctions. Such classes of weak equivalences arise naturally in homotopy theory. Examples include the class of functors for which the induced map on nerves is a weak homotopy equivalence and the class of functors for which the induced map on nerves is an $E$-(co)homology isomorphism for some spectrum $E$.

Let $\mathcal{W}_{s}$ be the weak equivalence structure on $T$ - $\mathcal{A l g}_{s}$ created by

$$
U_{s}: T-\mathcal{A l g}_{s} \rightarrow \text { Cat }
$$

and let $\omega$ be any of $l$, op, or ps. Then, by the appropriate variant of Theorem 2.8, the category $T-\mathcal{A l}_{g_{\omega}}$ has the weak equivalence structure created by $Q \dashv i$ and we have an adjoint equivalence of homotopy theories. The hypothesis that $\mathcal{V}$ contains all adjunctions ensures that the counit $\varepsilon$ is a weak equivalence. By Theorem 1.14 this is also the weak equivalence structure created by the forgetful functor $U_{\omega}: T-\mathcal{A l g}_{\omega} \rightarrow$ Cat.

Notation 2.10. Let we denote the class of weak homotopy equivalences in Cat, i.e., those functors which induce a weak homotopy equivalence on nerves. We abusively use this notation for any class of weak equivalences created by a functor to (Cat, we).

Example 2.11 (Symmetric monoidal categories). The prototypical example of this kind is when $T$ is the 2 -monad for symmetric monoidal categories. Then $T$ - $\mathcal{A l g}_{s}$ is the 2-category of symmetric monoidal categories, symmetric strict monoidal functors, and monoidal transformations, while $T$ - $\mathcal{A l g}_{l}$ has the same objects but symmetric lax monoidal functors. Let $\mathcal{V}=$ we and let the underlying category functor SymMonCat ${ }_{s} \rightarrow$ Cat create weak equivalences. By Theorem 2.8 we have the following adjoint equivalence 
of homotopy theories over (Cat,we).

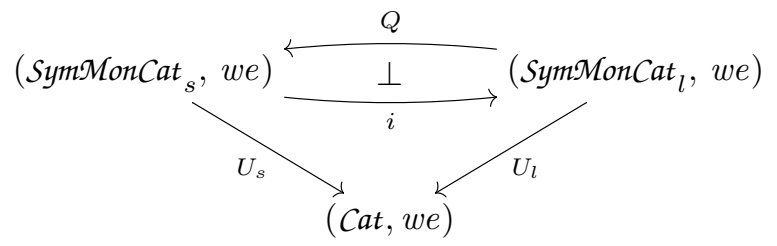

As noted above, one also has pseudo and oplax variants of this example which likewise give adjoint equivalences of homotopy theories. The pseudo algebra maps in this case are the strong symmetric monoidal maps.

Example 2.12 (Symmetric monoidal categories and normal functors). A slight variant of our first example uses a different 2-monad $T$ on $\mathrm{Cat}_{*}$ whose algebras are still symmetric monoidal categories. In this case, the specified base point becomes the unit object of the symmetric monoidal structure. The category $T$ - $\mathcal{A l g}_{s}$ consists of symmetric monoidal categories and symmetric strict monoidal functors, while $T$ - $\mathcal{A l g}_{l}$ is now the category of symmetric monoidal categories and normal (i.e., strictly unit preserving) symmetric lax monoidal functors. We take $\mathcal{V}=w e$ in $\mathrm{Cat}_{*}$ to be the class of unbased weak homotopy equivalences (created by the forgetful functor to Cat). By Theorem 2.8 we have the following adjoint equivalence of homotopy theories over $\left(\mathrm{Cat}_{*}, \mathrm{we}\right)$.

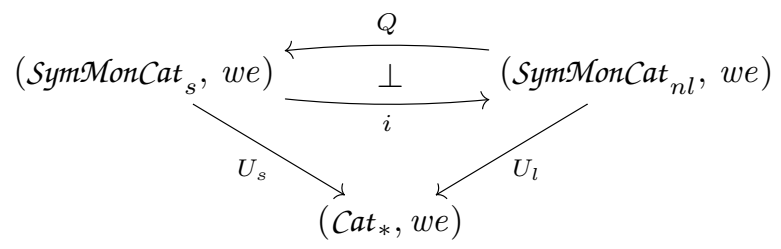

We also have oplax and pseudo variants of the previous examples. Combining these yields the following strengthening of [Man10, 3.9].

Theorem 2.13. The homotopy theory of (SymMonCat ${ }_{s}$, we) is equivalent to the homotopy theory of each of the following.

- $\left(\right.$ SymMonCat $_{p s}$, we)

- $\left(\right.$ SymMonCat $_{l}$, we $)$

- $\left(\right.$ SymMonCat $_{\text {op }}$, we)
- $\left(\right.$ SymMonCat $_{n p s}$, we)

- $\left(\right.$ SymMonCat $_{n l}$, we)

- $\left(\right.$ SymMonCat $_{n o p}$, we)

Example 2.14 (Stable equivalences of symmetric monoidal categories). For a final variant concerning symmetric monoidal categories, we take the normal, oplax version of the above example. For the "underlying" category we now take the category of $\Gamma$-categories, with $\mathcal{V}=s t e q$ being the class of stable equivalences [BF78]. This example differs from the previous ones in that we do not know whether $K$ satisfies monadicity and therefore cannot apply Theorem 2.8. However we can apply Theorems 1.11 and 1.14 directly. Let $U_{\lambda}=K$ be the $K$-theory functor for normal, oplax symmetric monoidal functors from [Man10], and let $U_{\tau}=K$ be the restriction to strict functors. 
Let $\mathcal{W}_{s}=s t e q$ be the weak equivalences created by $K$. Then the left adjoint $Q$ arises as in the previous examples but via the oplax variant of Theorem 2.4. We therefore have the following adjoint equivalence of homotopy theories over $(\Gamma-$ Cat st $e q)$.

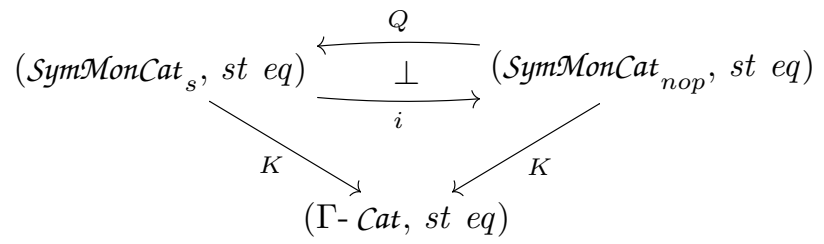

Once again we can consider other variants, making use of the alternative definitions of $K$ given in [Man10]. Together these give the following generalization of [Tho95, Man10].

Theorem 2.15. The homotopy theory of (SymMonCat ${ }_{s}$, st eq) is equivalent to the homotopy theory of each of the following.

- (SymMonCat $_{\text {ps }}$, st eq)

- $\left(\right.$ SymMonCat $_{l}$, st eq)

- $\left(\right.$ SymMonCat $_{\text {op }}$, st eq)
- $\left(\right.$ SymMonCat $_{n p s}$, st eq)

- $\left(\right.$ SymMonCat $_{n l}$, st eq)

- $\left(\right.$ SymMonCat $\left._{n o p}, s t e q\right)$

Our next examples concern $n$-fold monoidal categories for $n \geqslant 1$. These were introduced by Balteanu-Fiedorowicz-Schwänzl-Vogt [BFSV03] and are the algebras over an operad $\mathscr{M}_{n}$ whose geometric realization is equivalent to the little $n$-cubes operad.

Alternatively, an $n$-fold monoidal category can be defined iteratively as a monoid in the category $(n-1)-\mathcal{M}_{\text {on }}$ Cat $_{n l}$ of $(n-1)$-fold monoidal categories and normal lax monoidal maps. Laxity of the monoid structure map gives rise to interchange maps between the $n$ different monoidal products and also to a hexagonal interchange axiom. A 1-fold monoidal category is simply a monoidal category. The notion of braided monoidal category is equivalent to that of a 2 -fold monoidal category where both products are the same and their interchange transformation is invertible.

To apply our general theory we must identify the lax maps of $n$-fold monoidal categories as the lax algebra maps for the 2-monad associated to $\mathscr{M}_{n}$. This does not appear in the literature, but follows from Bourke's 2-dimensional monadicity (see Section 3).

Proposition 2.16. Let $n \geqslant 1$. The 2-category $n$-MonCatl of $n$-fold monoidal categories and lax maps is 2-equivalent to the 2-category of algebras and lax algebra maps associated to the 2-monad $\mathscr{M}_{n}$.

We prove Proposition 2.16 in Section 3.2.

Example 2.17 (Iterated monoidal categories). Let $T$ be the 2-monad on Cat associated to the operad $\mathscr{M}_{n}$. Let $\mathcal{V}=$ we and let $\mathcal{W}_{s}$ be created by the forgetful functor. By Proposition 2.16 and Theorem 2.8 we have the following adjoint equivalence of 
homotopy theories over (Cat, we).

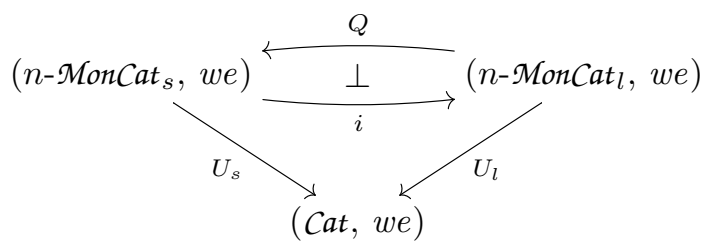

One also has a normal version of the previous example, similar to Example 2.12. The lax algebra maps in this case correspond to the $n$-fold monoidal category maps considered in [BFSV03].

Example 2.18 (Group completion equivalences of iterated monoidal categories). Let $\mathcal{V}=g c e q$ be the class of weak equivalences upon group completion of nerves. For $n=1$ this means equivalence after applying $\Omega B$ as discussed in [Seg74, BFSV03]. Applying Theorem 1.11 we have the following adjoint equivalence of homotopy theories.

$$
\left(n-\mathcal{M o n C a t}_{s}, g c \text { eq) } \underset{\frac{Q}{i}}{\stackrel{\perp}{i}}\left(n-\mathcal{M o n C a t}_{l}, g c \text { eq }\right)\right.
$$

\subsection{Diagrams in a 2-category}

Let $I$ be a small 2-category and let $\mathcal{K}$ be a complete and cocomplete 2-category. Let $\mathrm{ob} I$ denote the discrete 2-category with the same objects as $I$. The inclusion $\mathrm{ob} I \rightarrow I$ induces a 2 -functor

$$
U:[I, \mathcal{K}] \rightarrow[\mathrm{ob} I, \mathcal{K}]
$$

where $[-,-]$ denotes the 2-category of 2-functors, 2-natural transformations, and modifications.

This $U$ has left and right adjoints given by Kan extensions and is conservative so is 2-monadic. The associated 2 -monad $T=U \circ$ Lan can be computed using a coend formula. Now since $U$ has both adjoints it preserves all limits and colimits. The left Kan extension is a left adjoint so preserves colimits. Therefore $T$ preserves all colimits and hence is accessible. We summarize this discussion in Proposition 2.19.

Proposition 2.19. The 2-functor

$$
U:[I, \mathcal{K}] \hookrightarrow[\mathrm{ob} I, \mathcal{K}]
$$

is 2-monadic and the associated 2-monad is accessible.

The next result recognizes $[I, \mathcal{K}]_{l}$ as the 2-category of algebras and lax maps over $T$.

Proposition 2.20. Let $I, \mathcal{K}$, and $T$ be as above. Then $T$ - $\mathcal{A l g}_{l} \cong[I, \mathcal{K}]_{l}$, the 2-category of diagrams, lax transformations, and modifications.

We prove Proposition 2.20 simultaneously with a reduced version, Proposition 2.27, using Bourke's 2-dimensional monadicity in Section 3.2. One also has pseudo and oplax versions of Proposition 2.20 giving $T-\mathcal{A} \mathscr{L}_{p s} \cong[I, \mathcal{K}]_{p s}$ and $T-\mathcal{A l g}_{o p} \cong[I, \mathcal{K}]_{o p}$. These facts are well-known in the 2-categorical literature [BKP89, Example 6.6] and 
follow from a straightforward, if uninteresting, calculation. The reduced version does not appear in the literature to our knowledge, but is the case of interest for topological applications.

Our next examples concern categories with group actions. Let $G$ be a discrete group, and let $I=B G$ be the small category with one object having automorphisms given by $G$ and let $\mathcal{K}=$ Cat. A diagram $B G \rightarrow$ Cat is precisely a category with a $G$-action, and strict diagram maps are $G$-equivariant functors. For any morphism variant $\omega$ we denote $G-$ Cat $_{\omega}=[B G, \mathrm{Cat}]_{\omega}$. Note that $[\mathrm{obBG}, \mathrm{Cat}]$ is Cat.

The pseudonatural maps are functors that preserve equivariance only up to coherent natural isomorphisms. These appear naturally in examples, for instance, in Merling's study of equivariant algebraic $K$-theory [Mer17]. There are several notions of weak equivalence in the category of small $G$-categories and strict equivariant maps that are of interest to algebraic topologists and we discuss these below.

Example 2.21 ( $G$-categories with underlying weak equivalences). In this example we consider $G$ - Cat with weak equivalences being the equivariant functors that are weak homotopy equivalences on underlying categories. Let we denote this class. Combining Propositions 2.19 and 2.20 with Theorem 2.8, we have the following adjoint equivalence of homotopy theories over (Cat, we).

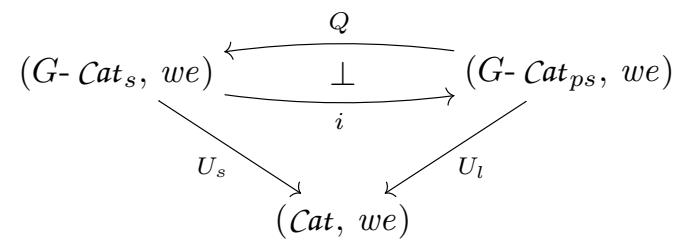

Non-example 2.22 ( $G$-categories with $G$-weak equivalences). Let $G$-we denote the class of $G$-weak equivalences, i.e., the equivariant functors $F: \mathcal{C} \rightarrow \mathcal{D}$ that induce weak homotopy equivalences on fixed points $\mathrm{C}^{H} \rightarrow \mathcal{D}^{H}$ for all subgroups $H$ of $G$ [May96, $\mathrm{BMO}^{+}$15]. These are the weak equivalences of primary homotopical interest.

The counit of the adjunction

$$
G-\mathrm{Cat}_{s} \stackrel{Q}{\stackrel{\perp}{i}} G-\mathrm{Cat}_{p s}
$$

is not a $G$-weak equivalence unless $G$ is trivial. Indeed, for the terminal category * with trivial $G$-action, $Q(*)=E G$, the category with set of objects equal to $G$ and a unique morphism between any two objects. The action of $G$ on $E G$ is given by left multiplication. The counit $E G \rightarrow *$ is a non-equivariant weak homotopy equivalence but not a $G$-weak equivalence since $E G^{H}$ is the empty category for all nontrivial subgroups. This means that $Q \dashv i$ is not an adjoint equivalence of homotopy theories between $\left(G-C^{-} a t_{s}, G-w e\right)$ and $\left(G-C a t_{p s}, \mathcal{W}_{p s}\right)$ for any choice of $\mathcal{W}_{p s}$.

\subsection{Reduced diagrams in a 2-category}

Note. We remind the reader that all limits and colimits are to be interpreted in the Cat-enriched sense. Thus a terminal object $*$ in $\mathcal{K}$ is one such that $\mathcal{K}(x, *)$ is the terminal category for all objects $x$. 
In this section, let $I$ be a small 2 -category with a zero object 0 and let $\mathcal{K}$ be a complete and cocomplete 2 -category with terminal object $*$.

Definition 2.23. A reduced diagram is a 2 -functor $X: I \rightarrow \mathcal{K}$ such that $X(0) \cong *$.

Let $[I, \mathcal{K}]_{\text {red }}$ denote the 2-category of reduced 2-functors, all 2-natural transformations, and modifications. Let

$$
j:[I, \mathcal{K}]_{\mathrm{red}} \hookrightarrow[I, \mathcal{K}]
$$

denote the inclusion of reduced diagrams into all diagrams. We define

$$
R:[I, \mathcal{K}] \rightarrow[I, \mathcal{K}]_{\text {red }}
$$

using a quotient construction as follows. If $X$ is any diagram and $a \in I$, define $R X(a)$ by the pushout below.

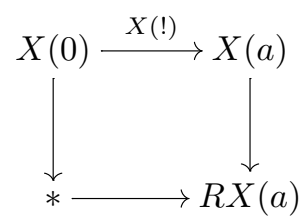

This levelwise pushout is a pushout in the 2-category $[I, \mathcal{K}]$ as we now explain. Let $c_{0} X$ be the constant diagram on $X(0)$. The maps $X(0 \rightarrow a)$ for $a \in I$ are the components of a map of diagrams $c_{0} X \rightarrow X$. The diagram $R X$ is then the pushout below.

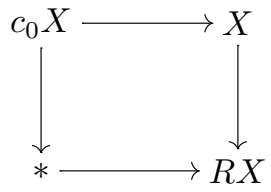

This construction is reduced because a pushout along an isomorphism is an isomorphism. The universal property of the 2-categorical pushout shows that $R$ is a 2 -functor $[I, \mathcal{K}] \rightarrow[I, \mathcal{K}]_{\text {red }}$

Proposition 2.24. The construction $R$ above is left 2-adjoint to the inclusion

$$
j:[I, \mathcal{K}]_{\text {red }} \hookrightarrow[I, \mathcal{K}] .
$$

Proof. For any diagram $X$ we have 1-cells

$$
X(a) \rightarrow R X(a)
$$

which form a 2-natural transformation

$$
\eta_{X}: X \rightarrow j R X
$$

by the 2-dimensional nature of the universal property of the pushout. As $X$ varies in $[I, \mathcal{K}]$, these assemble into a 2-natural transformation $\eta$ from the identity to $j R$.

If $Y$ is reduced, these 1-cells are isomorphisms because each pushout along the isomorphism $Y(0) \cong *$ is an isomorphism. Their inverses give a 2-natural transformation

$$
\varepsilon_{Y}: R j Y \rightarrow Y,
$$

which will assemble into a 2-natural transformation from $R j$ to the identity 2-functor 
on $[I, \mathcal{K}]_{\text {red }}$. One of the triangle identities is immediate from the definition of $\varepsilon$. To prove the other, that

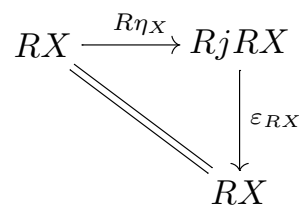

commutes, we prove that for an object $a$ of $I$, the 1-cells $\left(R \eta_{X}\right)_{a}$ and $\left(\eta_{R X}\right)_{a}$ are equal. Now $\left(R \eta_{X}\right)_{a}$ is defined as the unique dotted 1-cell that makes the diagram

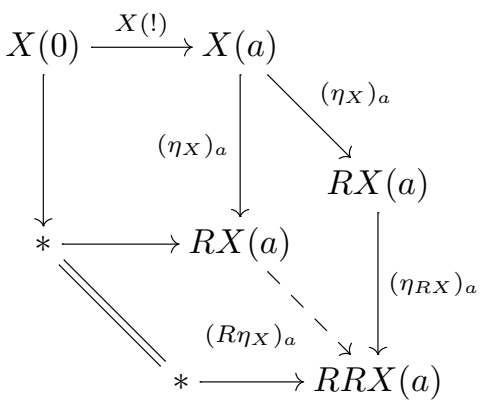

commute, but $\left(\eta_{R X}\right)_{a}$ is also such a morphism so $\left(R \eta_{X}\right)_{a}=\left(\eta_{R X}\right)_{a}$. This verifies the second triangle identity, since $\varepsilon$ was defined as the inverse of $\eta$.

Proposition 2.25. Let $\mathcal{K}$ be a complete and cocomplete 2-category. Then the inclusion

$$
j:[I, \mathcal{K}]_{\mathrm{red}} \hookrightarrow[I, \mathcal{K}]
$$

is 2-monadic and the associated 2-monad is accessible.

Proof. We apply the Cat-enriched version of Beck's monadicity theorem [Dub70]. To do this, we have only to check that $j$ is conservative, has a left adjoint, and preserves certain coequalizers. We have already constructed the left adjoint above. Isomorphisms of diagrams are levelwise isomorphisms in both categories, so $j$ is conservative. One can easily verify that coequalizers in $[I, \mathcal{K}]_{\text {red }}$ are computed levelwise, hence they exist and $j$ preserves all of them. The same is also true for filtered colimits (in fact all connected colimits), so $j$ preserves them and therefore the associated 2 -monad is accessible.

The 2-category $[I, \mathcal{K}]_{\text {red }}$ is also complete and cocomplete as a 2-category since it is a full reflective 2-category of a complete and cocomplete 2-category: limits are computed levelwise, and colimits are computed by first applying $j$, then taking the colimit in $[I, \mathcal{K}]$, and then applying $R$ to get a reduced diagram. For the remainder of this section we let $U$ denote the composition of $j$ with pullback along the inclusion $\mathrm{ob} I \hookrightarrow I$. The same arguments as above prove the next result.

Proposition 2.26. The composite

$$
U:[I, \mathcal{K}]_{\mathrm{red}} \hookrightarrow[I, \mathcal{K}] \hookrightarrow[\mathrm{ob} I, \mathcal{K}]
$$

is 2-monadic and the associated 2-monad is accessible. 
Let $T$ be the composite $U \circ R \circ$ Lan, the 2-monad associated with the composite adjunction. Then $T-\mathcal{A l g}_{s}$ is $[I, \mathcal{K}]_{\mathrm{red}}$.

Proposition 2.27. The 2-category $T$ - $\mathcal{A l g}_{l}$ is $[I, \mathcal{K}]_{\mathrm{red}, l}$, the 2-category of reduced diagrams, lax transformations, and modifications.

We prove Proposition 2.27 using Bourke's 2-dimensional monadicity in Section 3.2.

Example 2.28 ( $\Gamma$-objects and levelwise weak equivalences). Let $I$ be a skeleton of the category of finite based sets considered as a discrete 2-category and let $\mathcal{K}$ be either Cat or $2 \mathrm{Cat}_{2}$, the 2-category of 2-categories, 2-functors, and 2-natural transformations. Then $[I, \mathrm{Cat}]_{\mathrm{red}}$ is the 2-category of $\Gamma$-categories, $\Gamma$-functors, and $\Gamma$ transformations $[\mathrm{Seg} 74]$, while $\left[I, 2 \mathrm{Cat}_{2}\right]_{\mathrm{red}}$ is the 2-category of $\Gamma$-2-categories, $\Gamma$ 2 -functors, and $\Gamma$-transformations studied in [GJO17].

By Propositions 2.20 and 2.27, these are 2-monadic over $[\mathrm{obI}, \mathrm{Cat}]=[\mathbb{N}, \mathrm{Cat}]$ and, respectively, $\left[\mathbb{N}, 2 \mathrm{Cat}_{2}\right]$. The corresponding 2-categories with lax algebra maps are, respectively, $[I, \mathrm{Cat}]_{\mathrm{red}, l}$ and $\left[I, 2 \mathrm{Cat}_{2}\right]_{\mathrm{red}, l}$. These are the 2-categories of $\Gamma$-(2-)categories, $\Gamma$-lax (2-)functors, and $\Gamma$-transformations. Since the 2 -monads for these are accessible, we get a 2-adjunction $Q \dashv i$ as in Proposition 2.7. The counit of this adjunction is a right map as in Proposition 2.7 (2), and in particular a levelwise left adjoint.

Let $\mathcal{V}=w e$ be the class of levelwise weak homotopy equivalences, created by the forgetful functors to $[\mathbb{N}$, Cat $]$ and $\left[\mathbb{N}, 2\right.$ Cat $\left._{2}\right]$, respectively. Being a levelwise adjoint, the counit is a levelwise equivalence, so by Theorems 1.11 and 1.14 we have the following adjoint equivalences of homotopy theories.
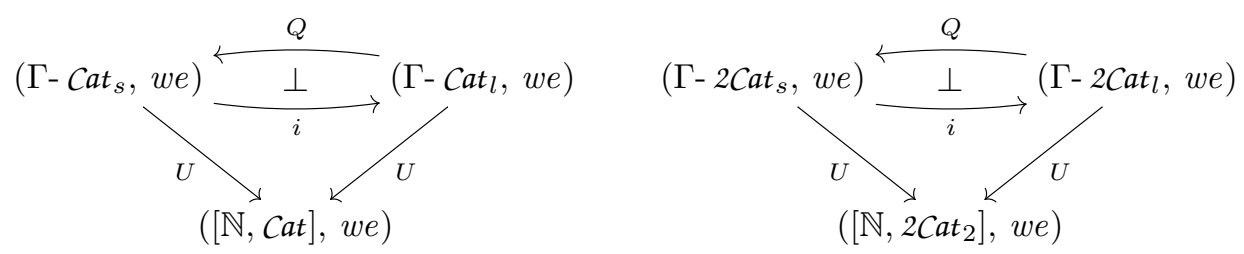

Note that this is a stronger result than what we were able to achieve using direct methods in [GJO17], namely it strengthens Theorem 4.37 and Corollary 4.47 of loc. cit. to equivalences of homotopy theories rather than just of homotopy categories.

Example 2.29 ( $\Gamma$-objects and stable equivalences). We can also consider the class of stable equivalences, $\mathcal{V}=s t e q$, which are now created by the levelwise nerve functor to $\Gamma$-sSet [BF78]. The counit of the adjunction between the categories with strict and lax maps is a stable equivalence since, once again, it is a levelwise equivalence. By Theorem 1.11 we have the following adjoint equivalences of homotopy theories.
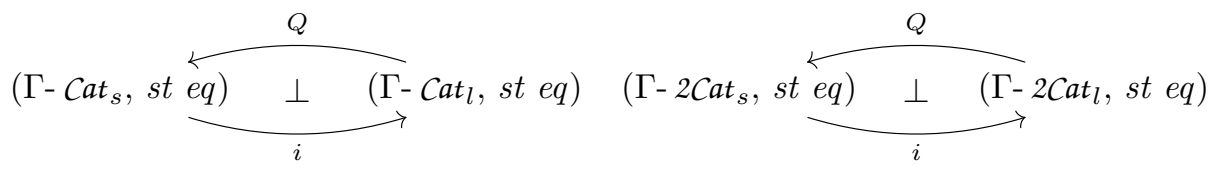

This is a strengthening of [GJO17, Corollary 4.49]. 


\section{2-dimensional monadicity}

In this section we recall and apply the 2-dimensional monadicity of Bourke [Bou14]. This goes beyond elementary Cat-enriched monadicity as it accounts simultaneously for both strict and lax algebra maps. This enables us to identify the bare-handed notions of lax morphisms as the lax algebra morphisms for iterated monoidal categories (Examples 2.17 and 2.18) and for $\Gamma$-(2-)categories (Examples 2.28 and 2.29).

\subsection{The 2-dimensional monadicity theorem for lax maps}

Throughout this section, let $\mathcal{B}$ be a 2-category and let $j: \mathcal{A}_{\tau} \rightarrow \mathcal{A}_{\lambda}$ be a 2-functor over $\mathcal{B}$ via 2 -functors $H_{\tau}: \mathcal{A}_{\tau} \rightarrow \mathcal{B}$ and $H_{\lambda}: \mathcal{A}_{\tau} \rightarrow \mathcal{B}$ as below.

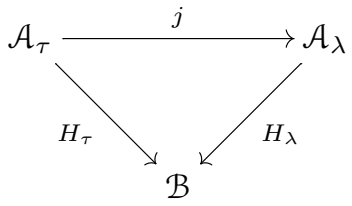

We further assume that $j$ is:

1. the identity on objects,

2. locally full and faithful on 2-cells, and

3. faithful on 1-cells.

In particular $j$ induces a map extension on underlying 1-categories. This is the notion of $\mathcal{F}$-category introduced in [LS12]. We will often suppress the subscripts on $H$ as they are clear from context.

Remark 3.2. For the remainder of this section we restrict to considering the strict/lax case. Analogous versions of the theory for strict/pseudo and for strict/oplax can be found in [Bou14].

Definition 3.3 (strict/lax monadic). We say that the pair $\left(H_{\tau}, H_{\lambda}\right)$ is strict/lax monadic if there are 2-equivalences

$$
\begin{aligned}
\mathcal{A}_{\tau} & \simeq T-\mathcal{A l g}_{s} \\
\mathcal{A}_{\lambda} & \simeq T-\mathcal{A l g}_{l}
\end{aligned}
$$

over $\mathcal{B}$ for some 2 -monad $T$ on $\mathcal{B}$ such that

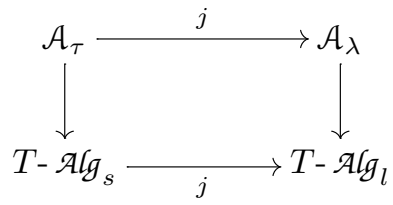

commutes.

Conditions for a given pair to be strict/lax monadic will be given below, and rely on the following definitions. Note that we have suppressed the inclusion $j$ in what follows. 
Definition 3.4 (Colax limit). Given $f: A \rightarrow B$ in $\mathcal{A}_{\lambda}$, the colax limit of $f$ consists of 1-cells $p_{f}$ and $q_{f}$ in $\mathcal{A}_{\tau}$ and a 2-cell $\sigma_{f}$ in $\mathcal{A}_{\lambda}$

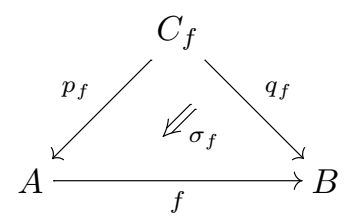

such that the following conditions hold.

1. Given 1-cells $r: X \rightarrow A, s: X \rightarrow B$ in $\mathcal{A}_{\lambda}$ and a 2-cell $\alpha: s \Rightarrow f r$ in $\mathcal{A}_{\lambda}$ as shown below, there is a unique $t \in \mathcal{A}_{\lambda}$ giving the indicated equalities.

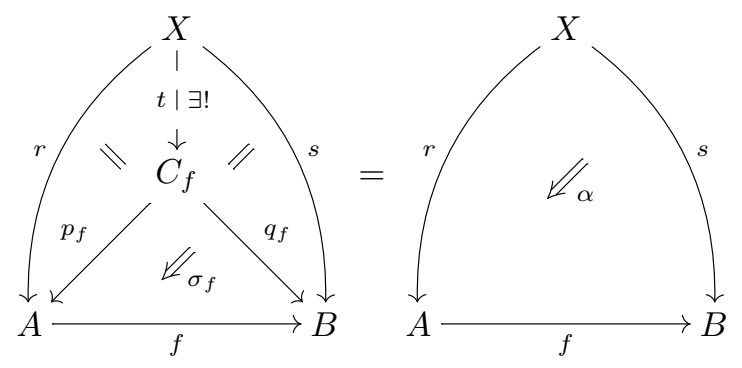

2. Let $(r, s, \alpha)$ and $\left(r^{\prime}, s^{\prime}, \alpha^{\prime}\right)$ be as above and let $\theta_{r}: r \Rightarrow r^{\prime}, \theta_{s}: s \Rightarrow s^{\prime}$ be such that $\alpha^{\prime} \theta_{s}=\left(f * \theta_{r}\right) \alpha$. Then there is a unique $\theta_{t}: t \Rightarrow t^{\prime}$ such that $p_{f} * \theta_{t}=\theta_{r}$ and $q_{f} * \theta_{t}=\theta_{s}$.

3. The structure 1-cell $t$ is in $\mathcal{A}_{\tau}$ if and only if $r$ and $s$ are both in $\mathcal{A}_{\tau}$.

We say that $j$ admits colax limits of arrows if the colax limit exists for every arrow $f$ in $\mathcal{A}_{\lambda}$.

Remark 3.5. We can also consider the colax limit of a morphism in a mere 2-category $\mathcal{A}$, in which case $\mathcal{A}=\mathcal{A}_{\tau}=\mathcal{A}_{\lambda}$ in the above definition and the third condition becomes vacuous. We would then say that $\mathcal{A}$ admits colax limits of arrows.

Definition 3.6 (Lax doctrinal adjunction). We say that the pair $\left(H_{\tau}, H_{\lambda}\right)$ satisfies lax doctrinal adjunction if given $f: A \rightarrow B$ in $A_{\tau}$ and an adjunction $\left(H_{\tau} f \dashv g, \varepsilon, \eta\right)$ in $\mathcal{B}$, there is a unique adjunction $(f \dashv \bar{g}, \bar{\varepsilon}, \bar{\eta})$ in $A_{\lambda}$ such that $H_{\lambda}(f \dashv \bar{g}, \bar{\varepsilon}, \bar{\eta})=\left(H_{\tau} f \dashv\right.$ $g, \varepsilon, \eta)$.

Theorem 3.7 ([Bou14]). Let $j: \mathcal{A}_{\tau} \rightarrow \mathcal{A}_{\lambda}$ be a 2-functor over $\mathcal{B}$ as in (3.1). Now suppose the following:

1. $H_{\tau}$ is 2-monadic with associated 2-monad $T$;

2. $j$ admits colax limits of arrows in $\mathcal{A}_{\lambda}$;

3. $\mathcal{B}$ admits colax limits of arrows;

4. $H_{\lambda}$ is locally faithful and reflects identity 2-cells; and

5. $\left(H_{\tau}, H_{\lambda}\right)$ satisfies lax doctrinal adjunction.

Then $\left(H_{\tau}, H_{\lambda}\right)$ is strict/lax monadic with associated 2-monad T. 
Our goal is often to identify the lax morphisms without explicitly computing them. Theorem 3.7 accomplishes this by identifying the lax morphisms as the 1-cells of $\mathcal{A}_{\lambda}$, possibly up to a 2-equivalence of 2-categories.

\subsection{Applications of 2-dimensional monadicity}

We now give the proofs of Propositions 2.16, 2.20 and 2.27.

Proof of Proposition 2.16. We apply Theorem 3.7. Let $\mathcal{A}_{\tau}$ and $\mathcal{A}_{\lambda}$, respectively, be the 2-categories of $n$-fold monoidal categories with strict, respectively lax, maps and $n$-fold monoidal transformations. Let $\mathcal{B}=$ Cat , let $H_{\tau}$ and $H_{\lambda}$ be the respective forgetful functors and let $j$ be the inclusion. The only conditions which are not immediate are that $\left(H_{\tau}, H_{\lambda}\right)$ satisfies lax doctrinal adjunction and that $j$ admits colax limits of lax arrows. However both are straightforward to verify, as we now sketch.

To show that $\left(H_{\tau}, H_{\lambda}\right)$ satisfies lax doctrinal adjunction, suppose that $f: A \rightarrow B$ is a strict map of $n$-fold monoidal categories and that $g: B \rightarrow A$ is an adjoint to the underlying functor of categories. Then one can construct a lax monoidal structure map for $g$ via the following composite

$$
g(b) \otimes_{i} g\left(b^{\prime}\right) \stackrel{\eta}{\rightarrow} g f\left(g(b) \otimes_{i} g\left(b^{\prime}\right)\right)=g\left(f g(b) \otimes_{i} f g\left(b^{\prime}\right)\right) \stackrel{g\left(\varepsilon \otimes_{i} \varepsilon\right)}{\longrightarrow} g\left(b \otimes_{i} b^{\prime}\right) .
$$

This is an instance of doctrinal adjunction for $\otimes_{i}[$ Kel74]. One uses the strict structure of $f$ and the triangle identities to verify these lax monoidal structures are compatible with the interchange transformations.

To show that $j$ admits colax limits of lax maps, one constructs the colax limit of underlying categories and verifies that it is endowed with an $n$-fold monoidal structure. If $f: A \rightarrow B$ is a lax map of $n$-fold monoidal categories then the colax limit in Cat is a category $C$ whose objects are triples $\left(a, b, \sigma_{b, a}\right)$ where $a \in A, b \in B$, and $\sigma_{b, a}: b \rightarrow f(a)$ is a morphism in $B$. The morphisms of $C$ consist of pairs of morphisms between the component objects such that the obvious squares in $B$ commute. For each index $i$, the $i$ th monoidal product on $C$ is determined componentwise by the $i$ th monoidal products on $A$ and $B$ and the lax monoidal structure maps for $f$. The interchange maps are given pairwise by those in $A$ and $B$. The compatibility of $f$ with interchange ensures that this defines a valid interchange for $C$. Verification of the necessary axioms consists of routine diagram algebra which we omit for the sake of brevity.

Proofs of Propositions 2.20 and 2.2\%. We apply Theorem 3.7. Let $\mathcal{A}_{\tau}$ be either $[I, \mathcal{K}]_{\text {red }}$ or $[I, \mathcal{K}]$ and let $\mathcal{B}=[\mathrm{ob} I, \mathcal{K}]$ and let $H_{\tau}$ be the map induced by the inclusion $\mathrm{ob} I \hookrightarrow I$. Let $\mathcal{A}_{\lambda}$ be the category of reduced or, respectively, unreduced diagrams with lax transformations, and let $j: \mathcal{A}_{\tau} \hookrightarrow \mathcal{A}_{\lambda}$ be the inclusion.

We now verify the five conditions of Theorem 3.7. Condition (1) is proved in Propositions 2.19 and 2.26. Condition (3) follows because $I$ is small and $\mathcal{K}$ is cocomplete. Condition (2) is straightforward by computing colax limits levelwise; i.e., in $\mathcal{B}=[\mathrm{ob} I, \mathcal{K}]$ and verifying that these extend to a 2 -functor on $I$. The 2-dimensional aspect of the universal property for the levelwise colax limit ensures the universal property of the colax limit in $\mathcal{A}_{\lambda}$. 
We verify condition (4) in the unreduced case, noting that this immediately implies the same condition for the reduced case. The functor $H_{\lambda}$ is the forgetful functor $[I, \mathcal{K}]_{l} \rightarrow[\mathrm{ob} I, \mathcal{K}]$. This is clearly locally faithful and reflects identity 2-cells.

For condition (5), let $f: X \rightarrow Y$ be a 2-natural transformation of diagrams on $I$ and let $f_{a} \dashv g_{a}$ be an adjunction for each $a \in \mathrm{ob} I$. We construct a lax transformation $\bar{g}$ in the following way. For $r: a \rightarrow b$ in $I$, define a 2 -cell $\bar{g}_{r}: X(r) g_{a} \Rightarrow g_{b} Y(r)$ as the composite

$$
X(r) g_{a} \stackrel{\eta_{b} 11}{\Longrightarrow} g_{b} f_{b} X(r) g_{a}=g_{b} Y(r) f_{a} g_{a} \stackrel{11 \varepsilon_{a}}{\Longrightarrow} g_{b} Y(r),
$$

where $\eta$, respectively $\varepsilon$, are the unit, respectively counit, for the object-wise adjunction between $H_{\lambda} f$ and $g$. The middle equality is given by the strict naturality of $f$. To see that $\bar{g}$ satisfies the axioms of a lax transformation one uses the triangle identities and 2-naturality of $f$. Now $(f, \bar{g}, \varepsilon, \eta)$ gives the unique adjunction lifting the object-wise adjunction and this completes the verification of (5).

\section{References}

[BF78] A.K. Bousfield and E.M. Friedlander, Homotopy theory of $\Gamma$-spaces, spectra, and bisimplicial sets, Geometric Applications of Homotopy Theory II (Proc. Conf., Evanston, Ill., 1977), Lecture Notes in Math., vol. 658, Springer, Berlin, 1978, pp. 80-130.

[BFSV03] C. Balteanu, Z. Fiedorowicz, R. Schwänzl, and R. Vogt, Iterated monoidal categories, Adv. Math. 176 (2003), no. 2, 277-349. doi:10.1016/S0001-8708(03)00065-3

[BG16a] J. Bourke and R. Garner, Algebraic weak factorisation systems I: Accessible AWFS, J. Pure Appl. Algebra 220 (2016), no. 1, 108-147. doi:10.1016/j.jpaa.2015.06.002

[BG16b] _ Algebraic weak factorisation systems II: Categories of weak maps, J. Pure Appl. Algebra 220 (2016), no. 1, 148-174. doi:10.1016/j.jpaa.2015.06.003

[BK12] C. Barwick and D.M. Kan, A characterization of simplicial localization functors and a discussion of DK equivalences, Indag. Math. (N.S.) 23 (2012), nos. 1-2, 69-79. doi:10.1016/j.indag.2011.10.001

[BKP89] R. Blackwell, G. Kelly, and A. Power, Two-dimensional monad theory, J. Pure Appl. Algebra 59 (1989), no. 1, 1-41 (English). doi:10.1016/0022-4049(89)90160-6

$\left[\mathrm{BMO}^{+} \mathbf{1 5}\right]$ A.M. Bohmann, K. Mazur, A. Osorno, V. Ozornova, K. Ponto, and C. Yarnall, A model structure on GCat, Women in Topology: Collaborations in Homotopy Theory, Contemp. Math., vol. 641, Amer. Math. Soc., Providence, RI, 2015, pp. 123-134.

[Bou14] J. Bourke, Two-dimensional monadicity, Adv. Math. 252 (2014), 708747. doi:10.1016/j.aim.2013.11.007

[Cra95] S.E. Crans, Quillen closed model structures for sheaves, J. Pure Appl. Algebra 101 (1995), no. 1, 35-57. doi:10.1016/0022-4049(94)00033-F 
[DK80] W.G. Dwyer and D.M. Kan, Simplicial localizations of categories, J. Pure Appl. Algebra 17 (1980), no. 3, 267-284. doi:10.1016/0022-4049(80)90049-3

[Dub70] E.J. Dubuc, Kan Extensions in Enriched Category Theory, Lecture Notes in Math., vol. 145, Springer-Verlag, Berlin-New York, 1970.

[EM06] A.D. Elmendorf and M.A. Mandell, Rings, modules, and algebras in infinite loop space theory, Adv. Math. 205 (2006), no. 1, 163-228. doi:10.1016/j.aim.2005.07.007

[GJO17] N. Gurski, N. Johnson, and A. M. Osorno, K-theory for 2-categories, Adv. Math. (2017), To appear. arXiv:1503.07824

[GT06] M. Grandis and W. Tholen, Natural weak factorization systems, Arch. Math. (Brno) 42 (2006), no. 4, 397-408 (English).

[Kel74] G. Kelly, Doctrinal adjunction, Category Seminar (Proc. Sem., Sydney 1972/1973), Lecture Notes Math., vol. 420, Springer, Berlin, 1974, pp. 257-280.

[KS74] G.M. Kelly and R. Street, Review of the elements of 2-categories, Category Seminar (Proc. Sem., Sydney, 1972/1973), Lecture Notes in Math., vol. 420, Springer, Berlin, 1974, pp. 75-103.

[LS12] S. Lack and M. Shulman, Enhanced 2-categories and limits for lax morphisms, Adv. Math. 229 (2012), no. 1, 294-356. doi:10.1016/j.aim.2011.08.014

[Man10] M.A. Mandell, An inverse K-theory functor, Doc. Math. 15 (2010), 765-791.

[May74] J.P. May, $E_{\infty}$ spaces, group completions, and permutative categories, New Developments in Topology (Proc. Sympos. Algebraic Topology, Oxford, 1972), London Math. Soc. Lecture Note Ser., vol. 11, Cambridge Univ. Press, London, 1974, pp. 61-93.

[May96]_, Equivariant Homotopy and Cohomology Theory, CBMS Regional Conference Series in Mathematics, vol. 91, Published for the Conference Board of the Mathematical Sciences, Washington, DC; by the American Mathematical Society, Providence, RI, 1996. With contributions by M. Cole, G. Comezaña, S. Costenoble, A. D. Elmendorf, J. P. C. Greenlees, L. G. Lewis, Jr., R. J. Piacenza, G. Triantafillou, and S. Waner.

[May09]_ The Construction of $E_{\infty}$ Ring Spaces from Bipermutative Categories, Geom. Topol. Monogr., vol. 16, Mathematical Sciences Publishers, March 2009.

[Mer17] M. Merling, Equivariant algebraic K-theory, 2015. Mathematische Zeitschrift (2017), vol. 285, issue 3-4, pp. 1205-1248. doi:10.1007/s00209-016-1745-3

[Oso12] A. Osorno, Spectra associated to symmetric monoidal bicategories, Algebr. Geom. Topol.12 (2012), no. 1, 307-342. doi:10.2140/agt.2012.12.307 
[Qui73] D. Quillen, Higher algebraic K-theory. I, Algebraic K-Theory I, (Proc. Conf., Battelle Inst., 1972), Lecture Notes Math., vol. 341, 1973, pp. 85147. doi:10.1007/BFb0067053

[Rez01] C. Rezk, A model for the homotopy theory of homotopy theory, Trans. Amer. Math. Soc. 353 (2001), no. 3, 973-1007 (electronic). doi:10.1090/S0002-9947-00-02653-2

[Seg74] G. Segal, Categories and cohomology theories, Topology 13 (1974), 293-312. doi:10.1016/0040-9383(74)90022-6

[Sta71] J.D. Stasheff, $H$-spaces and classifying spaces: foundations and recent developments, Algebraic Topology (Univ. Wisconsin, Madison, Wis., 1970), Proc. Sympos. Pure Math., vol. XXII, Amer. Math. Soc., Providence, R.I., 1971, pp. 247-272.

[Tho95] R.W. Thomason, Symmetric monoidal categories model all connective spectra, Theory Appl. Categ. 1 (1995), no. 5, 78-118 (electronic).

[Wal85] F. Waldhausen, Algebraic K-theory of spaces, Algebraic and Geometric Topology (New Brunswick, N.J., 1983), Lecture Notes in Math., vol. 1126, Springer, Berlin, 1985, pp. 318-419. doi:10.1007/BFb0074449

Nick Gurski nick.gurski@case.edu

Department of Mathematics, Applied Mathematics, and Statistics, Case Western Reserve University, Yost Hall, 2049 Martin Luther King Jr. Drive, Cleveland, OH 44106-7058, USA

http://mathstats.case.edu/faculty/nick-gurski/

Niles Johnson niles@math.osu.edu

Department of Mathematics, The Ohio State University Newark, 1179 University Drive, Newark, OH 43055-1766, USA

http://nilesjohnson.net

Angélica M. Osorno aosorno@reed.edu

Department of Mathematics, 3203 SE Woodstock Blvd, Portland, OR 97202-8199, USA

http://people.reed.edu/ aosorno/ 